\title{
Investigation of a small force standard with the mass based method
}

\author{
Gang $\mathrm{Hu}^{1}$, Jile Jiang ${ }^{1}$, Zhimin Zhang ${ }^{1}$, Yue Zhang ${ }^{1}$, Uwe Brand ${ }^{2}$, Min-Seok Kim ${ }^{3}$ \\ ${ }^{1}$ National Institute of Metrology, No.18, Bei San Huan Dong Lu, 100029 Chao Yang District, Beijing, P.R.China \\ ${ }^{2}$ Physikalisch-Technische Bundesanstalt (PTB), 38116 Braunschweig, Germany \\ ${ }^{3}$ Korea Research Institute of Standards and Science (KRISS), Daejeon, Republic of Korea
}

\begin{abstract}
A small force standard with the mass based method is developed. It is composed of an electromagnetic compensation balance, a nano-positioning stage and a displacement and pitch angle adjustment unit. The small force standard is used to measure spring constants of cantilevers in Atomic Force Microscope (AFM) and forces of small force transducers in related applications. Since the balance and the nano-positioning stage are traced to mass and length standards, respectively, the small force standard is SI traceable. The principle and structure of the small force standard and its main components are discussed in detail. The spring constants of several cantilevers used in AFM were calibrated by the small force standard. The calibration results are demonstrated and uncertainties are evaluated. For verifying metrological characteristics of the small force standard, a preliminary international comparison between NIM, PTB and KRISS was carried out. The comparison results were in good agreement and indicate that the calibration capabilities of the participating small force standards were equivalent within their reported uncertainties.
\end{abstract}

\section{Section: RESEARCH PAPER}

Keywords: small force; electromagnetic compensation balance; nano-positioning stage; cantilevers; atomic force microscope

Citation: Gang Hu, Jile Jiang, Zhimin Zhang, Yue Zhang, Uwe Brand, Min-Seok Kim, Investigation of a small force standard with the mass based method, Acta IMEKO, vol. 6, no. 2, article 4, July 2017, identifier: IMEKO-ACTA-06 (2017)-02-04

Section Editor: Min-Seok Kim, Research Institute of Standards and Science, Korea

Received May 27, 2017; In final form May 27, 2017; Published July 2017

Copyright: (C) 2017 IMEKO. This is an open-access article distributed under the terms of the Creative Commons Attribution 3.0 License, which permits unrestricted use, distribution, and reproduction in any medium, provided the original author and source are credited

Funding: This work was supported by the Ministry of Science and Technology of China, China

Corresponding author: Gang Hu, e-mail: hugang@nim.ac.cn

\section{INTRODUCTION}

There is a great demand for small force measurements in a large variety of scientific research fields such as material science, micromechanics, physics, biology or medicine. As an important measurement tool, MEMS (Micro-Electro-Mechanical System)based micro-force transducers are widely used in micro-robot systems, micromanipulation and micro-assembly systems, micro tribology research, biomechanical research, tiny implantable surgery, single cell manipulation and scanning probe microscopy (SPM) [1]. Thin films are the most widely used materials in components of MEMS and microelectronics, and is the basis of devices of microelectronic and MOEMS (microoptoelectromechanical) systems [2]. Accurate measurements and traceability of small forces are required in determining the mechanical properties of thin films. In the research on antigen-antibody adhesion and DNA molecules tensile strength, the small force between cells or molecules minimum order of magnitude of up to $\mathrm{pN}$ - has an impact on complex biological processes [3].

Atomic force microscopy and instrumented indentation are two techniques that are widely used in material science and nano- and biomechanics [4]. Spring constants of the cantilevers in atomic force microscopes (AFM) and load scale factors (LSF) of capacitance sensors in nano-indentation instruments are calibrated based on different principles and methods. But most of them are not traceable to SI units. As a result, mechanical properties established from different manufacturers and devices often do not agree with each other. For example, Young's modulus measured by AFM using different cantilevers can differ by orders of magnitude [4]. These inconsistencies are hindering a successful commercialization of nano- and biotechnology. 
For solving the problem, some NMIs launched small force research projects and developed SI traceable standards over the last decade. Generally, there are two kinds of small force standards in the micro-newton to nano-newton range: the first one is based on the mass method, such as the micro-force measuring devices at PTB [5], and NFC (the nano force calibrator) at KRISS [6]; the second one is based on the electrostatic force realization principle, such as the EFB (electrostatic force balance) at NIST [7], the primary low force balance at NPL [8], and the force measurement system at CMS [9]. The establishment of these SI traceable standards significantly improves the reliability of small force measurement results. The first inter-laboratory comparison was carried out among KRISS, NIST, NPL and PTB from 2008 to 2010 [10]. The comparison results were in good agreement, suggesting that the calibration capabilities of the participating small force facilities were equivalent within their reported uncertainties.

NIM launched a small force metrology project in 2011 and developed two small force standards with afore-mentioned two principles [11]. In Section 2 the principle and structure of the small force standard with the mass based method are described. In the next two sections spring constant measurements of AFM cantilevers and uncertainty evaluation are presented. In Section 5 a preliminary international comparison between NIM, PTB and KRISS is introduced and its results are demonstrated. Finally, in the concluding section the structure and experimental results of the small force standard are summarized.

\section{THE PRINCIPLE AND STRUCTURE OF THE SMALL FORCE STANDARD}

The small force standard is composed of an electromagnetic compensation balance, a nano-positioning stage, and a displacement and pitch angle adjustment unit. Its structure and photo are shown in Figure 1. The cantilever to be calibrated is mounted on the nano-positioning stage and can move in vertical direction, driven by the stage. As soon as the cantilever is in contact with the weighing pan of the balance, it has an elastic deformation and applies an external force to the balance at same time. The external force is balanced by the electromagnetic force generated by the balance. The displacement of the nano-positioning stage is set up and controlled by a controller. At each displacement set point, the displacement of the stage, the output signals of the balance and cantilever are acquired simultaneously.

Generally, there are two kinds of small force sensors: passive and active types [5]. The cantilever is a commonly used passive sensor. The applied force can be converted into a deflection. The spring constant of the cantilever $k$ is calculated by the following equation:

$k=F / d$

where $F$ is the applied force, $d$ is the deflection of the cantilever, and $k$ is the spring constant of the cantilever.

The active sensor, which integrates strain gauges and a bridge circuit, converts the applied force into a voltage. The force sensitivity of sensor $S_{F}$ is given by

$$
S_{F}=F / \Delta U
$$

where $F$ is the applied force and $\Delta U$ is the output voltage change of the sensor.

The electromagnetic force of the balance is traceable to the mass standards. The displacement of the nano-positioning stage is measured by a capacitance sensor in the stage. The output voltage of the sensor can be measured by an electrical measuring instrument. The displacement sensor and the electrical measuring instrument can be traced to length and electrical standards, respectively. In these ways, SI traceability of $k$ and $S_{F}$ are realized.

The electromagnetic compensation balance is a key component in the small force standard. The XP6U type balance made by Mettler-Toledo is adopted. Its maximum capacity is $6.1 \mathrm{~g}$ and the readability is $0.1 \mu \mathrm{g}$. Correspondingly, the maximum force is about $60 \mathrm{mN}$, and the force resolution is about $1 \mathrm{nN}$. The schematic diagram is shown in Figure 2. The weighing pan is connected to a compensation coil through a coupling element and a lever mechanism. The compensation coil is put in a permanent magnet. When a force $F$ is applied to the weighing pan, lever and coil move together in vertical direction. Their displacements are measured by an embedded displacement sensor. The current in the coil increases through an automatic compensation circuit. Therefore, an electromagnetic force is generated in the coil. It is equal to the applied force in value and opposite in direction, and restores the weighing pan to its original position. The current in the coil is proportional to the applied force $F$.

The other key component is the nano-positioning stage. The P-621.1CD type piezo linear stage made by PI is adopted. Its closed-loop travel is $100 \mu \mathrm{m}$, the closed-loop resolution is 0.4 $\mathrm{nm}$, the closed-loop linearity error is $0.02 \%$. A high stiffness is achieved with the FEA-optimized design of the frictionless flexure elements, which assure excellent guiding accuracy and dynamics. As a result, its pitch/yaw is within $\pm 3 \mu \mathrm{rad}$. PI's proprietary capacitive sensor is used. It is free of friction and

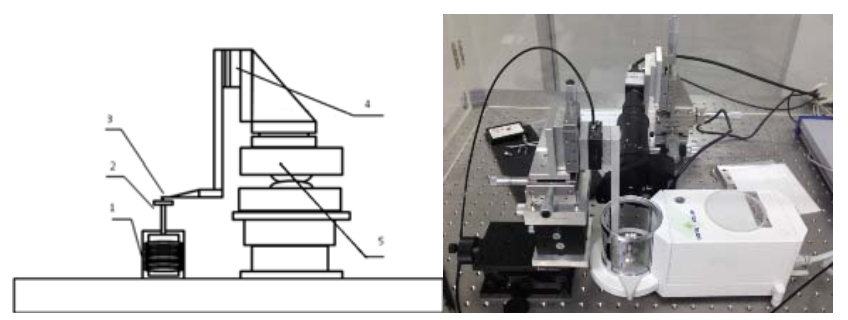

1 electromagnetic compensation balance 2 weighting pan 3 cantilever 4 nano-positioning stage

5 displacement and pitch angle adjustment unit

Figure 1. The structure and photo of NIM's small force standard with the mass based method.

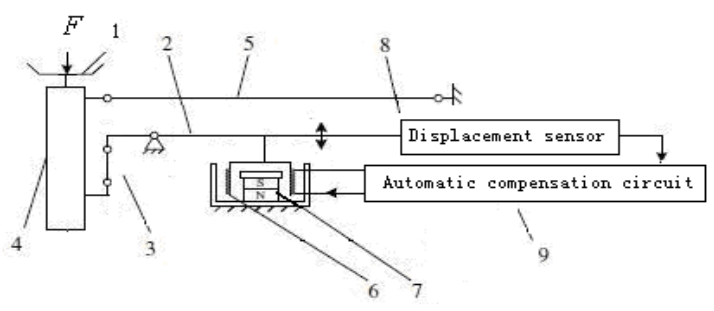

1 weighing pan 2 lever mechanism 3 coupling element 4 pan carrier 5 parallel guiding system 6 compensation coil 7 permanent magnet 8 displacement sensor 9 automatic compensation circuit

Figure 2. The schematic diagram of electromagnetic compensation balance. 
hysteresis and has a very high level of linearity.

The displacement and pitch angle adjustment units, which are composed of a three-dimensional linear slide and a twodimensional tilting stage, are used to adjust displacements and pitch angles of the cantilever in 5 degrees of freedom. Besides, a microscopic observation unit is applied to monitor the position and contact state of the cantilever and weighing pan. For minimizing the influence of vibration and air flow, the standard is put on a vibration isolation desk, and shielded by an organic glass enclosure to ensure a stable environmental condition.

\section{THE SPRING CONSTANT MEASUREMENTS AND CORRECTION}

\subsection{The spring constant measurements}

The spring constants of several cantilevers used in AFM were calibrated by the small force standard. The main specifications of the cantilevers are listed in Table 1. Two pieces of cantilevers for each type, which are numbered No.1 and No.2, respectively, were measured. For CLFC, there are 3 cantilevers with different lengths. The longest cantilever (length $400 \mu \mathrm{m}$ ) was used in our experiments.

A silicon plate is fixed on the weighing pan of the electromagnetic compensation balance for the measurement of cantilevers with tip (NSG03、CSG10). A conical diamond probe tip with $1 \mu \mathrm{m}$ tip radius and $90^{\circ}$ cone angle is applied to measure the tipless cantilevers (CLFC). The silicon plate and conical diamond probe tip are shown in Figure 3. The measurement position for NSG03、CSG10 is approximately at the centre of the cantilever tip. For CLFC, it is approximately at the end of the cantilever beam.

The measurement procedure is conducted as follows: 1. The cantilever is mounted on the small force standard. Adjust the displacement and pitch angle adjustment unit, make the cantilever to the proper position above the weighing pan and perpendicular to the balance axis (tilted angle is zero).

2. Drive the nano-positioning stage and make the cantilever move downward in vertical direction. As soon as a force is applied to the cantilever, the indication of the balance has a

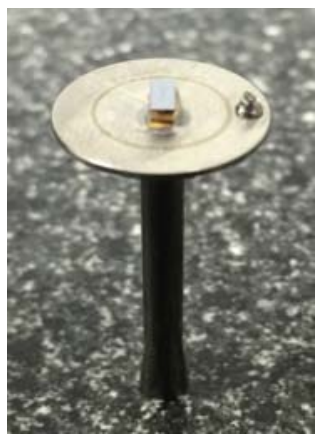

(a) The silicon plate

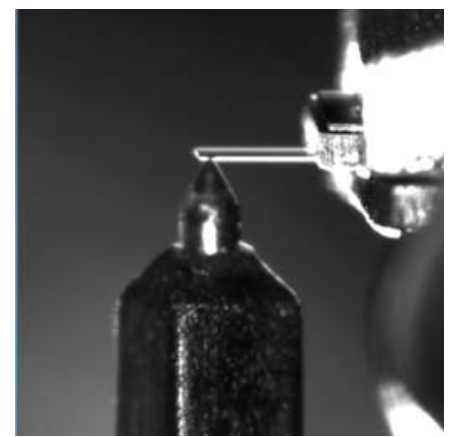

(b) conical diamond probe tip
Figure 3. The silicon plate and probe tip for different cantilevers.

significant increase. This position is determined as contact point.

3. The displacement control of the nano-positioning stage is adopted. The nano-positioning stage with cantilever moves downward step by step in vertical direction. The cantilever is loaded up to maximum deflection with 6 uniformly distributed points. Then it is unloaded down to the original deflection with the same 6 points. There is $30 \mathrm{~s}$ waiting time at each displacement set point. Once the output of the balance is stable, the indicated mass of the balance and displacement of the nano-positioning stage are acquired simultaneously. The whole experiment includes 10 cycles.

The force and displacement graphs are shown in Figure 4. The $\mathrm{x}$-coordinate indicates absolute displacements of the nanopositioning stage. The y-coordinate indicates corresponding forces calculated from the output of the balance. The red circles represent measurement points of the loading process and the blue crosses represent measurement points of the unloading process. The spring constants of the cantilevers are derived from a linear regression equation based on force-displacement data of loading and unloading.

The small force standard was operated under ambient conditions. The experiments were carried out in the range 21.0 ${ }^{\circ} \mathrm{C}$ to $22.0^{\circ} \mathrm{C}$.

Table 1. The main specifications of the cantilevers used in our experiments.

\begin{tabular}{|c|c|c|c|}
\hline Type & Dimensional parameters $(\boldsymbol{\mu m})$ & Spring constant (N/m) \\
\hline SSG03 & Length: 130 & Nominal value: $0.5-2.2$ \\
Typical value: 1.1
\end{tabular}



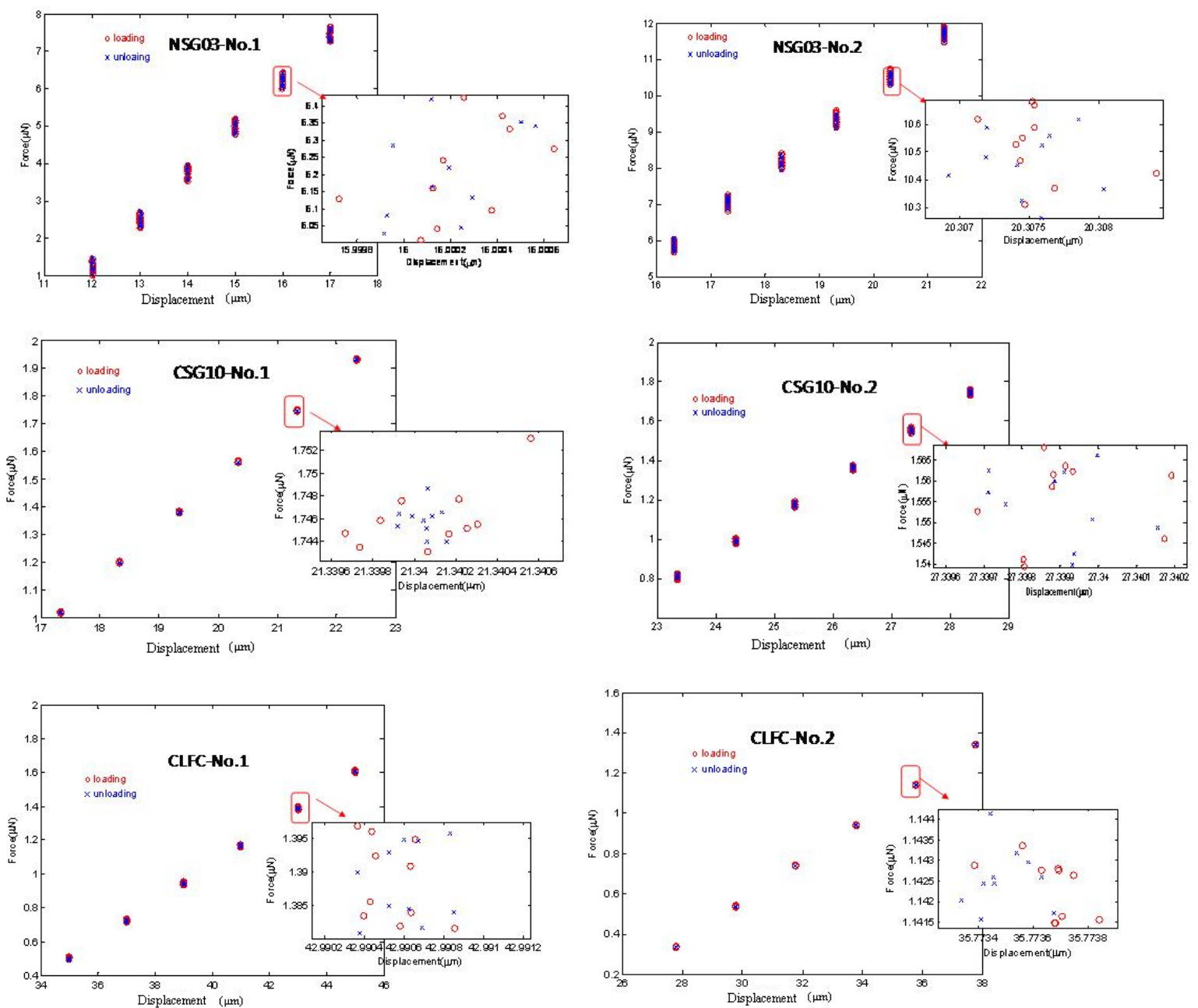

Figure 4. The force and displacement graphs of the spring constant measurements.

\subsection{The correction of the measurement results}

The cantilever, balance and stage are connected in series in the process of spring constant measurements. Considering the stiffness of the measurement chain, which includes the balance, the stage, and the displacement and pitch angle adjustment unit, the measurement results of the spring constants should be corrected.

The stiffness of the measurement chain was measured, while the cantilever was not mounted on the stage. The conical diamond probe tip, shown in Figure 3(b), was mounted on the weighing pan of the balance. The measurement procedure was conducted as shown in Figure 5. The displacement interval is 5 $\mathrm{nm}$, and the waiting time is $30 \mathrm{~s}$ at each displacement set point. The whole measurement has 10 cycles.

In calculating the deflection of the measurement chain, the deformation of the Si surface created by the diamond probe tip should be taken into account. The deformation $h$ is estimated by [12]

$h=\left(\frac{3}{4}\right)^{2 / 3} \cdot \frac{F^{2 / 3}}{E_{r}^{2 / 3} \cdot R^{1 / 3}}$

with

$\frac{1}{E_{r}}=\frac{1-v_{1}^{2}}{E_{1}}+\frac{1-v_{2}^{2}}{E_{2}}$ where $R$ is the radius of the diamond probe tip, $F$ is the applied force, $E_{\mathrm{r}}$ is the reduced Young's modulus, $E_{1}, E_{2}$ are the Young's modulus of diamond and silicon, $v_{1}, v_{2}$ are Poisson's ratios of diamond and silicon, respectively.

The deflection of the measurement chain is corrected by subtracting the deformation of the Si surface $b$ from the measured deflection. If a $100 \mu \mathrm{N}$ force is applied, there will be approximately $6.1 \mathrm{~nm}$ deformation according to (3).

The stiffness of the measurement chain is derived from a linear regression equation based on force-deflection data of loading and unloading. The measurement results are shown in Figure 6. The stiffness of the measurement chain $k_{\mathrm{i}}$ was determined as $1099.1 \mathrm{~N} / \mathrm{m}$ with a relative standard uncertainty $u\left(k_{i}\right) 6.3 \%$.

The measurement result of the spring constant $k_{m}$ is corrected by the following equation [10], [13]:

$k_{c}=\frac{k_{i} k_{m}}{k_{i}-k_{m}}$

where $k_{c}$ is the corrected spring constant.

The measurement results and the corrected results of the spring constants are summarized in Table2.

\section{THE UNCERTAINTY EVALUATION}

The uncertainty evaluation is based on the ISO/IEC Guide to the Expression of Uncertainty in Measurement. The 


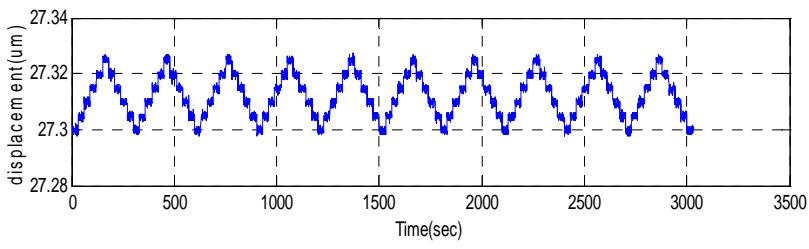

(a) Displacement graph

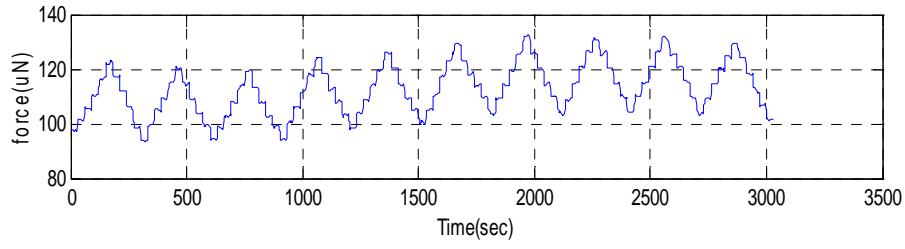

(b) Force graph

Figure 5. The displacement and force graph of the stiffness of the measurement chain.

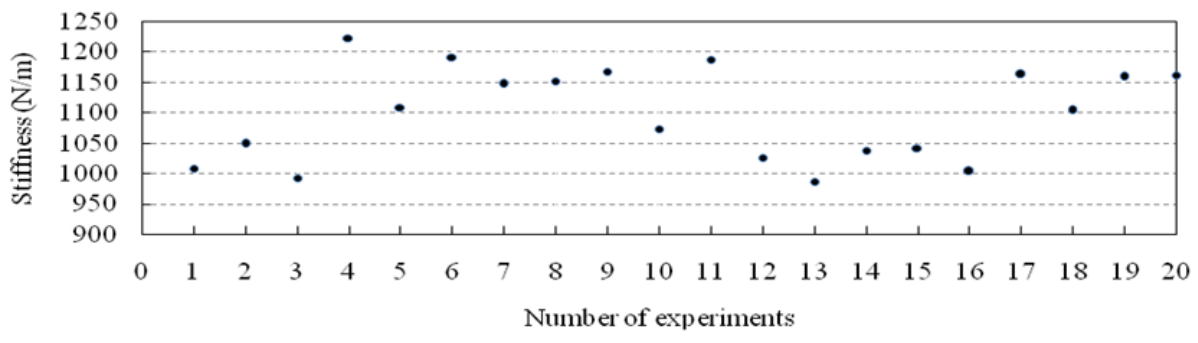

Figure 6. The measurement results of the stiffness of the measurement chain.

Table 2. The measurement results and the corrected results of the spring constants.

\begin{tabular}{|c|c|c|}
\hline Type/No. & The measurement results of spring constants $\boldsymbol{k}_{\boldsymbol{m}} \mathbf{( N / \mathbf { m } )}$ & The corrected results of spring constants $\boldsymbol{k}_{\boldsymbol{c}}$ (N/m) \\
\hline NSG03/ No.1 & $1.241 \mathrm{~N} / \mathrm{m}$ & $1.242 \mathrm{~N} / \mathrm{m}$ \\
\hline NSG03/ No.2 & $1.165 \mathrm{~N} / \mathrm{m}$ & $1.166 \mathrm{~N} / \mathrm{m}$ \\
\hline CSG10/ No.1 & $0.183 \mathrm{~N} / \mathrm{m}$ & $0.183 \mathrm{~N} / \mathrm{m}$ \\
\hline CSG10/ No.2 & $0.187 \mathrm{~N} / \mathrm{m}$ & $0.187 \mathrm{~N} / \mathrm{m}$ \\
\hline CLFC/ No.1 & $0.111 \mathrm{~N} / \mathrm{m}$ & $0.111 \mathrm{~N} / \mathrm{m}$ \\
\hline CLFC/ No.2 & $0.101 \mathrm{~N} / \mathrm{m}$ & $0.101 \mathrm{~N} / \mathrm{m}$ \\
\hline
\end{tabular}

uncertainty due to repeatability is classified as type A evaluation. The other uncertainty budgets are estimated as type B evaluation.

\subsection{The uncertainty evaluation of the force measurement}

For estimating the uncertainty due to the force measurement, the balance used in our small force standard was calibrated. In addition, the following factors, such as acceleration of gravity, ambient temperature change, measurement procedure, and the direction of the applied force, should be taken into consideration.

Two sets of standard weights with high accuracy were used for calibrating the balance. The measurement range runs from $50 \mu \mathrm{g}$ to $50 \mathrm{mg}$ with 10 calibration points - corresponding force range is approximately from $500 \mathrm{nN}$ to $500 \mu \mathrm{N}$. Standard weights of $1 \mathrm{mg}, 2 \mathrm{mg}, 5 \mathrm{mg}, 10 \mathrm{mg}, 20 \mathrm{mg}$, and $50 \mathrm{mg}$ with class $\mathrm{E}_{1}$ were used. Standard weights of $50 \mu \mathrm{g}, 100 \mu \mathrm{g}, 200 \mu \mathrm{g}$, $500 \mu \mathrm{g}$, made of aluminium alloy, were adopted and traced to the mass standard through an automatic mass comparator [14]. The calibration procedure of the balance refers to the Chinese verification regulation for electronic balances [15].

Generally, as the balance indication gets larger, the repeatability (absolute value) becomes larger. Therefore, the standard deviation of 10 times measurement results in $50 \mathrm{mg}$ ( absolute value) is considered as the uncertainty due to repeatability. The uncertainties due to standard weights are derived from their calibration results by the mass standard with higher class. The uncertainty due to the scale interval of the balance is estimated according to the guideline [16]. Since the position of the applied force is always in the centre of the weighing pan, the uncertainty due to eccentric load is negligible. The standard uncertainty due to the balance calibration $u_{\mathrm{f} 1}$ is determined by the root-squared sum of these uncertainty budgets.

The measured acceleration of gravity in the laboratory where the standard is built up amounts:

$g=9.801264562 \mathrm{~m} / \mathrm{s}^{2}, u_{\mathrm{f} 2}=3.0 \times 10^{-7}$.

The uncertainty due to ambient temperature changes $u_{\mathrm{f} 3}$, assuming a rectangular distribution, is calculated by

$u_{\mathrm{f} 3}=\frac{T_{\mathrm{C}} \Delta T}{2 \sqrt{3}}$

where the ambient temperature change in the laboratory $\triangle T$ is less than $1^{\circ} \mathrm{C}\left( \pm 0.5^{\circ} \mathrm{C}\right)$ and the temperature coefficient of the balance $T_{\mathrm{C}}$ is $0.0001 \% /{ }^{\circ} \mathrm{C}$. As a result, $u_{\mathrm{f} 3}=2.9 \times 10^{-7}$.

When the standard is used for calibration of the cantilever, the loading and unloading procedures are similar to those of the balance calibration. In this case, creep or hysteresis of the balance has a similar influence on the measurement results. The uncertainty due to differences of measurement procedures $u_{\mathrm{f} 4}$ is negligible.

Mounting of the cantilever may cause inconsistency of the directions between the applied force and gravity. The uncertainty due to this factor $u_{\mathrm{f} 5}$, assuming a projection distribution, is calculated as follows [17]: 
$u_{\mathrm{f} 5}=\frac{3(1-\cos \alpha)}{10}$

where $\alpha$ is the angle between the directions of the applied force and gravity, and generally is less than $1^{\circ}$. Therefore, $u_{\mathrm{f} 5}=$ $4.6 \times 10^{-5}$

The standard uncertainty budgets of the force measurement in each calibration point are summarized in Table 3.

The relative combined standard uncertainty of the force measurement $u(F)$ is calculated with the following equation:

$u(F)=\sqrt{u_{f 1}{ }^{2}+u_{f 2}{ }^{2}+u_{f 3}{ }^{2}+u_{f 4}{ }^{2}+u_{f 5}{ }^{2}}$

It is demonstrated in Table 3 that the force measurement uncertainty of the standard is better than $7.0 \times 10^{-3}$ in the range of $500 \mathrm{nN}-500 \mu \mathrm{N}$.

\subsection{The uncertainty evaluation of the spring constant measurement}

The uncertainty due to the repeatability $u_{1}$ is estimated by the standard deviation of the average of 10 measurement results.

For the type B evaluation, the following uncertainty contributions, such as force and displacement measurements, non-linearity of calibration curves, orientation of the cantilevers, location accuracy of the measurement point and the stiffness of the measurement chain should be taken into account.

The uncertainty due to the force measurement is estimated in 4.1. At smaller forces the uncertainty is larger. The uncertainty at the lower limit of the whole force range is considered as the uncertainty of the force measurement $u_{2}$.

The uncertainty due to the displacement measurement is estimated from the calibration results of the nano-positioning stage. The displacements of the nano-positioning stage were measured by a laser interferometer (Zygo ZMI 4004) over 100 $\mu \mathrm{m}$ travel range with $5 \mu \mathrm{m}$ step. The standard uncertainty of the measurement results was $68.2 \mathrm{~nm}$. A conservative estimation of the relative uncertainty due to the displacement measurement $u_{3}$ is determined as $1.36 \times 10^{-2}$.
Because the spring constant of the cantilever is derived from a linear regression equation of force-displacement data, the residuals of the linear regression are calculated and considered as the uncertainty due to the non-linearity of the calibration curves $u_{4}$.

The orientation of the cantilevers affects the measurement results. This factor is analyzed and discussed in the literature [6] and [13]. Assuming the maximum tilted angle is $1^{\circ}$, the relative uncertainty due to the orientation of the cantilever $u_{5}$ is $3.8 \times 10^{-3}$.

According to Euler's equation, the stiffness of the cantilever $k$ is related to Young's modulus and the cantilever dimensions [18]. Therefore, the location accuracy of the measurement point should be considered for tipless cantilevers. For cantilevers with tip (the measurement point is at the tip of the cantilever), the uncertainty due to location accuracy is negligible. In our standard, the location accuracy of the measurement point in the direction of the beam is estimated as $\pm 3 \mu \mathrm{m}$. Assuming a rectangular distribution, the relative standard uncertainty due to location accuracy $u_{6}$ can be given by [13]

$u_{6}=\sqrt{3}\left(\frac{\delta L}{L}\right)$

where $\delta L$ is the maximum position deviation from the ideal location. In the case of the CLFC, $u_{6}$ is calculated to be $1.3 \times$ $10^{-2}$ using $\delta L=3 \mu \mathrm{m}, L=400 \mu \mathrm{m}$.

The stiffness of the measurement chain $k_{\mathrm{b}}$ was determined to be $1099.1 \mathrm{~N} / \mathrm{m}$. Assuming a rectangular distribution, the relative standard uncertainty due to the stiffness of measurement chain $u_{7}$ is given by [13]

$u_{7}=\frac{1}{\sqrt{3}}\left|\frac{k_{m}}{k_{b-} k_{m}}\right| \frac{\delta k_{b}}{k_{b}}$

where $\delta k_{\mathrm{b}}=120 \mathrm{~N} / \mathrm{m}$ is the maximum deviation of the stiffness of the measurement chain $k_{\mathrm{b}}$.

The combined standard uncertainty $u\left(k_{c}\right)$ is determined using:

$u\left(k_{c}\right)=\sqrt{u_{1}^{2}+u_{2}^{2}+u_{3}^{2}+u_{4}^{2}+u_{5}^{2}+u_{6}^{2}+u_{7}^{2}}$

Table 3. Standard uncertainty budgets of the force measurement.

\begin{tabular}{|c|c|c|c|c|c|c|c|c|c|c|c|}
\hline \multirow{2}{*}{\multicolumn{2}{|c|}{ Standard uncertainty budgets }} & \multicolumn{10}{|c|}{ The measured forces $(\mu \mathrm{N})$} \\
\hline & & 0.5 & 1 & 2 & 5 & 10 & 20 & 50 & 100 & 200 & 500 \\
\hline \multirow{4}{*}{$\begin{array}{c}\text { Balance } \\
\text { calibration } \\
u_{\mathrm{f} 1}\end{array}$} & $\begin{array}{l}\text { Measurement } \\
\text { repeatability }\end{array}$ & $6.2 \times 10^{-3}$ & $3.1 \times 10^{-3}$ & $1.6 \times 10^{-3}$ & $6.2 \times 10^{-4}$ & $3.1 \times 10^{-4}$ & $1.6 \times 10^{-4}$ & $6.2 \times 10^{-5}$ & $3.1 \times 10^{-5}$ & $1.6 \times 10^{-5}$ & $6.2 \times 10^{-6}$ \\
\hline & $\begin{array}{l}\text { Standard } \\
\text { weights }\end{array}$ & $1.8 \times 10^{-3}$ & $1.2 \times 10^{-3}$ & $1.1 \times 10^{-3}$ & $6.7 \times 10^{-4}$ & $8.8 \times 10^{-4}$ & $4.4 \times 10^{-4}$ & $1.8 \times 10^{-4}$ & $8.8 \times 10^{-5}$ & $4.4 \times 10^{-5}$ & $2.3 \times 10^{-5}$ \\
\hline & $\begin{array}{l}\text { Scale interval } \\
\text { of the balance }\end{array}$ & $8.6 \times 10^{-4}$ & $4.3 \times 10^{-4}$ & $2.2 \times 10^{-4}$ & $8.6 \times 10^{-5}$ & $4.3 \times 10^{-5}$ & $2.2 \times 10^{-5}$ & $8.6 \times 10^{-6}$ & $4.3 \times 10^{-6}$ & $2.2 \times 10^{-6}$ & $8.6 \times 10^{-7}$ \\
\hline & Eccentric load & \multicolumn{10}{|c|}{ Negligible } \\
\hline \multicolumn{2}{|c|}{ The acceleration of gravity $u_{\mathrm{f} 2}$} & \multicolumn{10}{|c|}{$3.0 \times 10^{-7}$} \\
\hline \multicolumn{2}{|c|}{$\begin{array}{l}\text { Ambient temperature change } \\
\qquad u_{\mathrm{f} 3}\end{array}$} & \multicolumn{10}{|c|}{$2.9 \times 10^{-7}$} \\
\hline \multicolumn{2}{|c|}{$\begin{array}{l}\text { Difference of measurement } \\
\text { procedures } u_{\mathrm{f} 4}\end{array}$} & \multicolumn{10}{|c|}{ Negligible } \\
\hline \multicolumn{2}{|c|}{$\begin{array}{l}\text { Inconsistency of the } \\
\text { directions between applied } \\
\text { force and gravity } u_{\mathrm{f} 5}\end{array}$} & \multicolumn{10}{|c|}{$4.6 \times 10^{-5}$} \\
\hline \multicolumn{2}{|c|}{$\begin{array}{l}\text { Relative combined standard } \\
\text { uncertainty } u(F)\end{array}$} & $6.5 \times 10^{-3}$ & $3.4 \times 10^{-3}$ & $1.9 \times 10^{-3}$ & $9.2 \times 10^{-4}$ & $9.3 \times 10^{-4}$ & $4.7 \times 10^{-4}$ & $1.9 \times 10^{-4}$ & $1.0 \times 10^{-4}$ & $6.5 \times 10^{-5}$ & $5.2 \times 10^{-5}$ \\
\hline
\end{tabular}


Standard uncertainty budgets, the combined standard uncertainty and the standard uncertainty of the corrected spring constants are summarized in Table 4.

\section{PRELIMINARY INTERNATIONAL COMPARISON}

For verifying the metrological characteristics of the small force standard, a preliminary international comparison between NIM, PTB and KRISS was carried out in 2015. The small standards in PTB and KRISS, which are based on the electromagnetic compensation balance and a nano-positioning stage, were applied for this comparison [5], [6]. Three types of commercial cantilevers for AFM, which is mentioned in Section 3.1, were used in this comparison.

There was no identical measurement procedure in this comparison. The common aspects in the comparison are as follows:

1. The cantilevers were installed on the nano-positioning stage or the weighing pan of the balance in such a way that the plane of the cantilevers is perpendicular to the balance axis (tilted angle is zero).

2. The measurement range of deflections and the forces were roughly the same in different laboratories.

Uncertainty of measurement results is evaluated by each laboratory individually.

The deviations of measurement results between NIM and PTB/KRISS $\delta$ are calculated using:

$\delta=x_{\mathrm{NIM}}-x_{\mathrm{PTB} / \mathrm{KRISS}}$ where $x_{\mathrm{NIM}}, \chi_{\mathrm{PTB}} / \mathrm{KRISS}$ are the measurement results at NIM and PTB/KRISS, respectively.

The degree of agreement of the measurement results between NIM and PTB/KRISS is expressed by $E_{n}$. The ratio $E_{n}$ is given by

$E_{n}=\frac{\delta}{\sqrt{U_{\mathrm{NIM}}^{2}+U_{\mathrm{PTB} / \mathrm{KRISS}}^{2}}}$

where $U_{\mathrm{NIM}}, U_{\mathrm{PTB} / \mathrm{KRISS}}$ are the expanded uncertainties of the measurement results of NIM and PTB/KRISS, respectively.

The comparison results are shown in Table 5. This table shows that the absolute value of $E_{n}$ is less than 1 , the calibration capabilities of small force standards are equivalent within their reported uncertainties.

\section{CONCLUSIONS}

A small force standard with the mass based method is developed. It is composed of an electromagnetic compensation balance, a nano-positioning stage, and a displacement and pitch angle adjustment unit. The balance was calibrated by standard weights with high accuracy. A nano-positioning stage with good guiding accuracy is adopted and minimizes the influence of inconsistency of the directions between applied force and gravity. The displacement and pitch angle adjustment unit is used to adjust the displacement and pitch angle of the cantilever and improves the positioning accuracy. The spring constants of several cantilevers used in AFM were calibrated by the small force standard and uncertainties were evaluated.

Table4. Standard uncertainty budgets of the spring constant measurement.

\begin{tabular}{|c|c|c|c|c|c|c|}
\hline \multirow[b]{2}{*}{ Standard uncertainty budgets } & \multicolumn{6}{|c|}{ The relative standard uncertainty } \\
\hline & $\begin{array}{c}\text { NSG03 } \\
\text { No.1 }\end{array}$ & $\begin{array}{c}\text { NSG03 } \\
\text { No.2 }\end{array}$ & $\begin{array}{c}\text { CSG10 } \\
\text { No.1 }\end{array}$ & $\begin{array}{c}\text { CSG10 } \\
\text { No.2 }\end{array}$ & $\begin{array}{l}\text { CLFC } \\
\text { No.1 }\end{array}$ & $\begin{array}{l}\text { CLFC } \\
\text { No.2 }\end{array}$ \\
\hline Repeatability $u_{1}$ & $1.36 \times 10^{-3}$ & $1.90 \times 10^{-3}$ & $5.91 \times 10^{-4}$ & $5.79 \times 10^{-4}$ & $4.84 \times 10^{-4}$ & $3.13 \times 10^{-4}$ \\
\hline Force measurement $u_{2}$ & $3.40 \times 10^{-3}$ & $3.40 \times 10^{-3}$ & $3.40 \times 10^{-3}$ & $3.40 \times 10^{-3}$ & $3.40 \times 10^{-3}$ & $3.40 \times 10^{-3}$ \\
\hline Displacement measurement $u_{3}$ & $1.36 \times 10^{-2}$ & $1.36 \times 10^{-2}$ & $1.36 \times 10^{-2}$ & $1.36 \times 10^{-2}$ & $1.36 \times 10^{-2}$ & $1.36 \times 10^{-2}$ \\
\hline Non-linearity of calibration curve $u_{4}$ & $1.01 \times 10^{-2}$ & $1.08 \times 10^{-2}$ & $6.43 \times 10^{-3}$ & $7.43 \times 10^{-3}$ & $2.11 \times 10^{-3}$ & $1.73 \times 10^{-3}$ \\
\hline Orientation of the cantilever $u_{5}$ & $3.80 \times 10^{-3}$ & $3.80 \times 10^{-3}$ & $3.80 \times 10^{-3}$ & $3.80 \times 10^{-3}$ & $3.80 \times 10^{-3}$ & $3.80 \times 10^{-3}$ \\
\hline Location accuracy $u_{6}$ & negligible & negligible & negligible & negligible & $1.30 \times 10^{-2}$ & $1.30 \times 10^{-2}$ \\
\hline Stiffness of measurement chain $u_{7}$ & $7.12 \times 10^{-5}$ & $6.69 \times 10^{-5}$ & $1.05 \times 10^{-5}$ & $1.07 \times 10^{-5}$ & $6.36 \times 10^{-6}$ & $5.79 \times 10^{-6}$ \\
\hline $\begin{array}{l}\text { Relative combined standard uncertainty of } \\
\text { the corrected spring constants } u\left(k_{c}\right)\end{array}$ & $1.77 \times 10^{-2}$ & $1.82 \times 10^{-2}$ & $1.59 \times 10^{-2}$ & $1.63 \times 10^{-2}$ & $1.96 \times 10^{-2}$ & $1.96 \times 10^{-2}$ \\
\hline
\end{tabular}

Table 5. Comparison results.

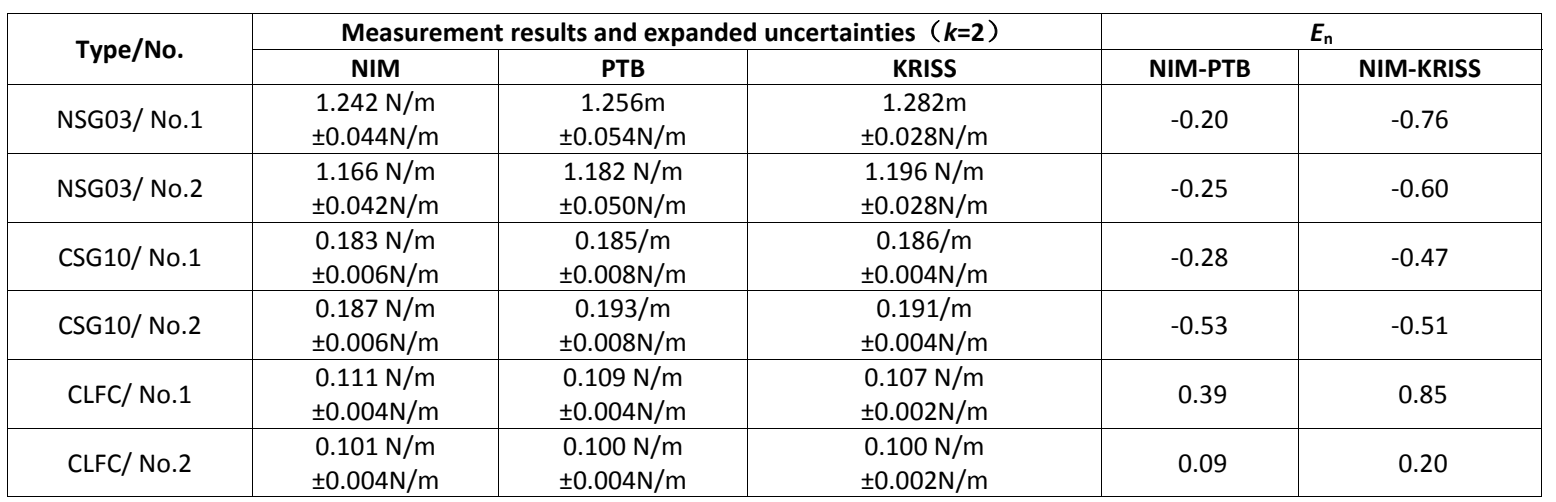


A preliminary international comparison between NIM, PTB and KRISS was carried out. The comparison results are in good agreement and indicate that the calibration capabilities of small force standards are equivalent within their reported uncertainties. It seems that there are systematic deviations in measurements of NSG03, CSG10 and CLFC, respectively. Further investigation and analysis on the sources of these systematic deviations will be made. Besides, the relative movement of the cantilever and other unknown phenomena at the contact point remains to investigate.

Structural improvement and optimization of NIM's small force standard will be made. Further metrological characteristics experiments and international comparisons with NMIs will be carried out. Better transfer standards should be applied, a more rigorous technical protocol should be discussed and determined for further comparisons.

\section{ACKNOWLEDGEMENT}

This work was supported by the National Key Technology Research and Development Program of the Ministry of Science and Technology of China under the project "Establishment of microgram mass standards and micro-nanonewton force standards and research on key technologies in traceability", project number: 2011BAK15B06.

\section{REFERENCES}

[1] Lu Xiaoguang, "Study on Microforce sensor based on piezoelectric thin film”, Master's degree thesis, Dalian University of Technology, 2007.

[2] Mei Tao, Kong Deyi, Zhang Peiqiang, Wu Xiaoping, "Mechanical properties and scaling effects in micro-electromechanical systems", Journal of Mechanical Strength, 23 (2001) pp. 373-379.

[3] Willemsen O H, Snel M M E, Cambi A, et al., "Biomolecular interactions measured by atomic force microscopy", Biophys, 79( 2000) pp.3267-3281.

[4] Min-Seok Kim, Jon R. Pratt, "SI traceability: Current status and future trends for forces below 10 microNewtons", Measurement, 43 (2010) pp.169-182.

[5] Ingo Behrens, Lutz Doering and Erwin Peiner, "Piezoresistive cantilever as portable micro force calibration standard", Journal of Micromechanics and Microengineering, 13 (2003) pp. S171S177.

[6] Min-Seok Kim, Jae-Hyuk Choi, Yon-Kyu Park and Jong-Ho Kim, "Atomic force microscope cantilever calibration device for quantified force metrology at micro- or nano-scale regime: the nano force calibrator (NFC)", METROLOGIA, 43 (2006) pp. 389-395.

[7] Jon R. Pratt, John A Kramar, David B Newell and Douglas T Smith, "Review of SI traceable force metrology for instrumented indentation and atomic force microscopy", Measurement Science and Technology, 16 (2005) pp. 2129-2137.

[8] Leach R, Chetwynd D, Blunt L, et al. "Recent advances in traceable nanoscale dimension and force metrology in the UK", Measurement Science and Technology, 17 (2006) pp. 467-476.

[9] Sheng-Jui Chen, Sheau-Shi Pan, Yu-Shan Yeh and Yi-Ching Lin, "Measurement of cantilever spring constant using an electrostatic sensing and actuating force measurement system", Measurement Science and Technology, 25 (2014) pp.1-10.

[10] Min-Seok Kim, Jon R. Pratt, Uwe Brand and Christopher W Jones, "Report on the first international comparison of small force facilities: a pilot study at the microNewton level", METROLOGIA, 49 (2012) pp.70-81.

[11] Hu gang, Song le, Meng feng, Zhang wei, Zhang zhimin, Zhang yue and Zheng yelong, "Research and development of small force standards at NIM" Measurement of Mass, Force and Torque (APMF 2013) International Journal of Modern Physics: Conference Series, 24 (2013) pp.1360020-1-9.

[12] T. Chudoba, N. Schwarzer, F. Richter, "Determination of elastic properties of thin films by indentation measurements with a spherical indenter", Surface and Coatings Technology, 127 (2000) pp.9-17.

[13] Min-Seok Kim, Jae-Hyuk Choi, Jong-Ho Kim and Yon-Kyu Park, "SI traceable determination of spring constants of various atomic force microscope cantilevers with a small uncertainty of 1\%", Measurement Science and Technology, 18 (2007), pp.3351-3358.

[14] Jian Wang, Peter Fuchs, Stefan Russi, et al, "Uncertainty Evaluation for System of Weighing Equations for the Determination of Microgram Weights", IEEE Transactions on Instrumentation And Measurements, 64( 8) (2015) pp. 22722279.

[15] Verification Regulation for Electronic Balance (JJG1036-2008), 2008.

[16] European co-operation for Accreditation (EA) Laboratory Committee, "EA-10/18 EA Guidelines on the calibration of non-automatic weighing instruments", 2005.

[17] Li qingzhong, Li yuhong, "Guideline to uncertainty evaluation of force, torque and hardness measurements", China Metrology Publishing House, Beijing, 2003, ISBN 7-5026-1758-2/TB ·649.

[18] M.-S. Kim, Y.-K. Park and J._H. Kim "Millimeter-Scale Piezoresistive Cantilevers for Accurate Force Measurements at the Nano-Newton Level", MAPAN-Journal of Metrology Society of India, 28(4) (2013) pp.251-257. 